\title{
Model Equations for Three-Dimensional Nonlinear Water Waves under Tangential Electric Field
}

\author{
Bo Tao \\ School of Architecture Engineering, Neijiang Normal University, Sichuan 641100, China \\ Correspondence should be addressed to Bo Tao; 275471543@qq.com
}

Received 18 May 2017; Revised 18 September 2017; Accepted 11 October 2017; Published 12 November 2017

Academic Editor: Prabir Daripa

Copyright (C) 2017 Bo Tao. This is an open access article distributed under the Creative Commons Attribution License, which permits unrestricted use, distribution, and reproduction in any medium, provided the original work is properly cited.

\begin{abstract}
We are concerned with gravity-capillary waves propagating on the surface of a three-dimensional electrified liquid sheet under a uniform electric field parallel to the undisturbed free surface. For simplicity, we make an assumption that the permittivity of the fluid is much larger than that of the upper-layer gas; hence, this two-layer problem is reduced to be a one-layer problem. In this paper, we propose model equations in the shallow-water regime based on the analysis of the Dirichlet-Neumann operator. The modified Benney-Luke equation and Kadomtsev-Petviashvili equation will be derived, and the truly three-dimensional fully localized traveling waves, which are known as "lumps" in the literature, are numerically computed in the Benney-Luke equation.
\end{abstract}

\section{Introduction}

Interfacial electrohydrodynamic waves have many important applications in mechanical, chemical, and electrical industries, such as electrospray ionization, cooling systems, coating process, and electrowetting (see [1-10] and the references therein). The electric stresses have great impact on electrified free surfaces or interfaces, which can not only modify wave patterns but also change stability characteristics of the system. These influences are of great practical interest since they can lead to quantity (e.g., heat or mass) transfer enhancement (see, e.g., $[5,11,12])$. The study of the stability of interfacial electrohydrodynamic waves was initiated by Melcher [3] and Taylor and McEwan [13], and the role of interfacial stresses resulting from electrodes was reviewed by Melcher and Taylor [4]. Recent theoretical research in this field has focused on the nonlinear phenomena and corresponding mechanisms, such as nonlinear coherent structures (e.g., [2, 5, 8, 10, 11, 1420]) and touchdown singularities (e.g., $[7,21])$. Considerable effects have been put into the modeling and numerical studies of nonlinear interfacial electrohydrodynamic waves.

For incompressible, inviscid, and irrotational flows, electrified interfacial waves were studied in the nonlinear regime under externally applied electric fields by different groups. The electric field is usually posed parallel or perpendicular to the undisturbed interface and is therefore called tangential or normal electric field, respectively. The orientation of the electric field with respect to the undisturbed interface plays an important role in the system. From the point of view of the linear theory, the dispersion relations are of the forms

$$
\begin{aligned}
& \omega^{2}=|k|\left(g+\frac{\sigma}{\rho} k^{2}\right)-\frac{E_{0}^{2} \beta\left(\epsilon_{p}\right)}{\rho} k^{2}, \\
& \omega^{2}=|k|\left(g+\frac{\sigma}{\rho} k^{2}\right)-\frac{E_{0}^{2} \gamma\left(\epsilon_{p}\right)}{\rho} k^{2},
\end{aligned}
$$

for two-dimensional gravity-capillary waves in deep water under normal and tangential electric field, respectively, where $\omega$ is the frequency, $k$ the wavenumber, $g$ the acceleration due to gravity, $\sigma$ the surface tension coefficient, $E_{0}$ the strength of the electric field, $\rho$ the density of the liquid, and $\epsilon_{p}$ the ratio of permittivities. The forms of functions $\beta(x)$ and $\gamma(x)$ depend on the specific problem; for example, $\beta(x)=x$ for normal electric field acting on a perfect conducting fluid $([2,8])$ and $\gamma(x)=(x-1)^{2} /(x+1)$ for a dielectric fluid under a tangential electric field ([10]). Linearly speaking, a tangential electric field provides a dispersive contribution so as to stabilize the system, while a considerably strong 
normal electric field can provide energy to a certain range of wavenumbers to induce instability. On the nonlinear side, Tilley et al. [7] proposed a nonlinear long-wave system to study the dynamics of electrified liquid sheets and found that the tangential electric field can delay the formation of the film rupture. More than that, Barannyk et al. showed in [15] that the tangential electric field can even suppress the RayleighTaylor instability in some situations. On the contrary, it can be deduced from the work by Gleeson et al. [16], Papageorgiou et al. [21], Lin et al. [2], and Wang [8] that the normal electric field has a destabilizing effect on the interface.

N. M. Zubarev and O. V. Zubareva $[10,20]$ and Tao and Guo [22] considered the electrified gas-fluid or vacuumfluid interface so that they can make the assumption that the permittivity of the fluid was much larger compared to that of gas (the permittivities for pure water and air are 80 and 1, resp.). As a consequence, the actual two-layer problem could be reduced to one layer, and the theoretical and numerical techniques developed for free-surface water wave problems can be generalized to include the electric field.

On the numerical side, most researches focus on periodic waves (see, e.g., $[5,7,10,14,21]$ ); however the studies of electrified solitary waves have begun recently. Easwaran [23] first derived the Korteweg-de Vries (KdV) equation in the context of electrohydrodynamic waves which admits sech ${ }^{2}$ soliton solutions naturally. Hammerton [17] studied solitary waves in the KdV-Benjamin-Ono equation, a model arising from a conducting liquid sheet under a normal electric field generated by two electrodes with a sufficiently large separation distance. Barannyk et al. [15] computed interfacial solitary waves based on a system describing long waves between two immiscible dielectric fluids. It is noted that $\mathrm{KdV}$ Benjamin-Ono equation was first derived in the context of interfacial waves between two immiscible fluids when surface tension is sufficiently strong (e.g., [24, 25]).

All the aforementioned papers confine themselves to two-dimensional (2D) problems, but there are relatively fewer studies on the three-dimensional (3D) case. Recent study on 3D electrohydrodynamic modeling has focused on conducting fluids under normal electric fields: Hunt et al. [18] proposed a one-way model with weak transverse variations which is called the Benjamin-Ono-Kadomtsev-Petviashvili equation, Aliev and Yurchenko [14] established a reduced system for the same setup, and Wang [8] developed fully nonlinear numerical models and weakly nonlinear theories for electrohydrodynamic surface waves in the Hamiltonian framework based on analyses of the Dirichlet-Neumann operators. In this paper, we are concerned with a dielectric liquid under a tangential electric field, and restoring forces due to gravity, surface tension, and Maxwell stresses are all taken into account. We will propose weakly nonlinear models (including both unidirectional and bidirectional equations) under Zubarev's approximation and numerically search for truly 3D solitary waves (which is known as "lumps" in the literature) within these models.

The rest of the paper is structured as follows. In Section 2, the governing equations are described and simplified by making the assumption that the permittivity of the fluid is large. In Section 3, the Dirichlet-Neumann operator is

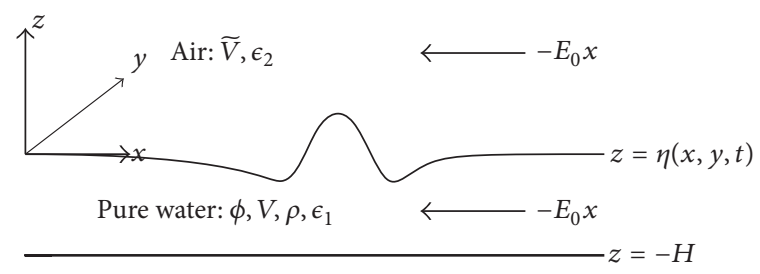

FIGURE 1: Schematic description of (pure) water-air interface in the presence of a horizontal electric field with uniform strength $-E_{0}$ in the far field.

introduced, which plays an important role in deriving weakly nonlinear models. In Section 4, a bidirectional model (the modified Benney-Luke equation) and a unidirectional model (the modified Kadomtsev-Petviashvili equation) are derived under the long-wave approximation. Then the main numerical results are followed, including the typical profiles of solitary waves, the bifurcation diagrams, and the formation of lumps resulting from the transverse instability of plane solitary waves.

\section{Formulation}

2.1. Governing Equations. We consider an incompressible, inviscid, and irrotational fluid, moving on a solid wall, under a horizontal electric field with uniform strength $-E_{0}$ in the far field. We introduce the Cartesian coordinates, such that $x$ and $y$ are horizontal variables, and $z$ points upwards with $z=0$ being the undisturbed air-water interface (see Figure 1). We denote the displacement of the air-water interface by $z=\eta(x, y, t)$; then the velocity potential of the fluid, which is denoted by $\phi$, is governed by

$$
\phi_{x x}+\phi_{y y}+\phi_{z z}=0 \text { for }-H<z<\eta(x, y, t) .
$$

And the voltage potentials within and above the fluid domain, denoted by $V$ and $\widetilde{V}$, respectively, are also governed by Laplace's equation:

$$
\begin{aligned}
& V_{x x}+V_{y y}+V_{z z}=0, \quad \text { for }-H<z<\eta(x, y, t), \\
& \widetilde{V}_{x x}+\widetilde{V}_{y y}+\widetilde{V}_{z z}=0, \text { for } z>\eta(x, y, t) .
\end{aligned}
$$

The kinematic and dynamic boundary conditions at the interface $z=\eta(x, y, t)$ are given by (see, e.g., $[10,22,26]$ )

$$
\begin{aligned}
\eta_{t}= & \phi_{z}-\nabla_{\perp} \eta \cdot \nabla_{\perp} \phi \\
\phi_{t}= & -\frac{1}{2}|\nabla \phi|^{2}+\frac{\epsilon_{p}-1}{8 \pi \rho} \nabla V \cdot \nabla \widetilde{V}-g \eta+\frac{\sigma}{\rho} \nabla_{\perp} \\
& \cdot\left[\frac{\nabla_{\perp} \eta}{\sqrt{1+\left|\nabla_{\perp} \eta\right|^{2}}}\right],
\end{aligned}
$$

where $\nabla=\left(\partial_{x}, \partial_{y}, \partial_{z}\right)^{\top}, \nabla_{\perp}=\left(\partial_{x}, \partial_{y}\right)^{\top}$, and $\epsilon_{p}$ is the ratio of the permittivity of water, $\epsilon_{1}$, to that of air, $\epsilon_{2}$. The detailed derivation of the electrostatic pressure at the interface between an ideal dielectric liquid and air (or vacuum) in 
the presence of free electric charges can be found in [26] or [5]. At the interface $z=\eta(x, y, t)$, the continuity condition of voltage potentials and the normal stress induced by electricity are, respectively,

$$
\begin{aligned}
V & =\widetilde{V} \\
\epsilon_{1}\left(V_{z}-\nabla_{\perp} \eta \cdot \nabla_{\perp} V\right) & =\epsilon_{2}\left(\widetilde{V}_{z}-\nabla_{\perp} \eta \cdot \nabla_{\perp} \widetilde{V}\right) .
\end{aligned}
$$

Following Barannyk et al. [15], the kinematic boundary conditions for $\phi$ and $V$ at $z=-H$ are of the same form

$$
\begin{gathered}
\phi_{z}=0, \\
V_{z}=0 .
\end{gathered}
$$

Finally, the far-field conditions for the electric field,

$$
\begin{aligned}
& \widetilde{V} \longrightarrow-E_{0} x, \text { for }|x|+|y| \longrightarrow \infty, \\
& V \longrightarrow-E_{0} x, \text { for }|x| \longrightarrow \infty,
\end{aligned}
$$

complete the whole system.

2.2. Simplification. In order to simplify the problem, we make assumption that the quantity $\epsilon_{2} / \epsilon_{1}$ is small. This is a reasonable assumption in many physical situations when the upper layer is occupied by air $\left(\epsilon_{2} \approx 1\right)$ and the lower layer is a fluid with much lager electric permittivity such as water $\left(\epsilon_{1} \approx 80\right)$. This assumption could decouple the system. We then nondimensionalize the system by choosing

$$
\left[\frac{H}{g}\right]^{1 / 2}, H, E_{0},\left[g H^{3}\right]^{1 / 2}
$$

as the time, length, voltage potential, and velocity potential scales, respectively. Therefore, following $[10,19,20]$ the dynamic boundary condition can be rewritten as

$$
\begin{aligned}
\phi_{t}= & -\frac{1}{2}|\nabla \phi|^{2}+\frac{E}{2}|\nabla V|^{2}-\eta+B \nabla_{\perp} \\
& \cdot\left[\frac{\nabla_{\perp} \eta}{\sqrt{1+\left|\nabla_{\perp} \eta\right|^{2}}}\right],
\end{aligned}
$$

where $E \triangleq \epsilon_{p} E_{0}^{2} / 4 \pi \rho g H^{3}$, and the rescaled surface tension coefficient becomes the Bond number $B \triangleq \sigma / \rho g H^{2}$. The rigid bottom is now represented by $z=-1$, and $V \rightarrow-x$ as $|x| \rightarrow \infty$. Meanwhile, the normal stress due to the electric field becomes

$$
V_{z}-\nabla_{\perp} \eta \cdot \nabla_{\perp} V=0 .
$$

Finally, we introduce the variable $W$ by $W \triangleq V+x$, and, as a consequence, $W \rightarrow 0$ as $|x| \rightarrow \infty$ and $W_{z}=0$ at $z=-1$. It also follows at the interface that

$$
\begin{aligned}
\phi_{t}= & -\frac{1}{2}|\nabla \phi|^{2}+\frac{E}{2}\left(|\nabla W|^{2}-2 W_{x}\right) \\
& -B \nabla_{\perp} \cdot\left[\frac{\nabla_{\perp} \eta}{\sqrt{1+\left|\nabla_{\perp} \eta\right|^{2}}}\right]
\end{aligned}
$$

$W_{z}-\nabla_{\perp} \eta \cdot \nabla_{\perp} W=-\eta_{x}$

\section{Dirichlet-Neumann Operators}

The Dirichlet-Neumann operator (DNO), which maps the Dirichlet boundary condition to normal derivatives on the boundary via solving Laplace's equation, plays an essential role in surface water wave problems. Coifman and Meyer [27] initially proved that if the Lipschitz-norm of wave profiles is smaller than a certain constant then DNO is analytic in two-dimensional problems. Subsequently, the analyticity of this operator was extended to three dimensions by Craig et al. [28], and Craig and Nicholls [29] and Nicholls and Reitich [30] delivered further results. If we define $\xi(x, y, t) \triangleq$ $\phi(x, y, \eta(x, y, t), t)$ as the surface velocity potential, the DNO, denoted by $G(\eta)$, can be defined by

$$
G(\eta) \xi=\phi_{z}-\nabla_{\perp} \eta \cdot \nabla_{\perp} \phi
$$

In the same vein, we define the surface voltage $\zeta(x, y, t) \triangleq$ $W(x, y, \eta(x, y, t))$. Since $W$ also satisfies the Laplace equation as well as the boundary condition $W_{z}=0$ at $z=-1$, as a consequence, we can use the same Dirichlet-Neumann operator to define

$$
G(\eta) \zeta=W_{z}-\nabla_{\perp} \eta \cdot \nabla_{\perp} W
$$

Using the boundary condition of the voltage potential (12), we have

$$
G(\eta) \zeta=-\eta_{x} \Longrightarrow \zeta=-G^{-1}(\eta) \partial_{x} \eta
$$

If we define a new pseudo-differential operator $\mathscr{P}(\eta) \triangleq$ $G^{-1}(\eta) \partial_{x}$, it follows that $\zeta=-\mathscr{P}(\eta) \eta$. Here we remark that strictly speaking $G^{-1} \partial_{x}$ is not a well-defined operator due to the singularity at the origin in the Fourier space; however, in the present problem $\mathscr{P}$ always appears with $\nabla_{\perp}$ (see (17) and (19)) which removes the singularity. We then reformulate the problem by use of the DNO. It is not difficult to conduct the following calculation:

$$
\begin{aligned}
& \phi_{z}-\eta_{x} \phi_{x}-\eta_{y} \phi_{y}=G(\eta) \xi, \\
& \phi_{x}+\eta_{x} \phi_{z}=\xi_{x}, \\
& \phi_{y}+\eta_{y} \phi_{z}=\xi_{y}, \\
& \Downarrow \\
& \phi_{x}=\frac{\left(1+\eta_{y}^{2}\right) \xi_{x}-\eta_{x} \eta_{y} \xi_{y}-\eta_{x} G(\eta) \xi}{1+\left|\nabla_{\perp} \eta\right|^{2}}, \\
& \phi_{y}=\frac{\left(1+\eta_{x}^{2}\right) \xi_{y}-\eta_{x} \eta_{y} \xi_{x}-\eta_{y} G(\eta) \xi}{1+\left|\nabla_{\perp} \eta\right|^{2}}, \\
& \phi_{z}=\frac{G(\eta) \xi+\nabla_{\perp} \eta \cdot \nabla_{\perp} \xi}{1+\left|\nabla_{\perp} \eta\right|^{2}} .
\end{aligned}
$$


Similarly, we can have

$$
\begin{gathered}
\eta_{x} W_{x}+\eta_{y} W_{y}-W_{z}=\eta_{x}, \\
W_{x}+\eta_{x} W_{z}=-\partial_{x} \mathscr{P}(\eta) \eta, \\
W_{y}+\eta_{y} W_{z}=-\partial_{y} \mathscr{P}(\eta) \eta, \\
\Downarrow \\
W_{x}=\frac{\eta_{x}^{2}+\eta_{x} \nabla_{\perp} \eta \cdot \nabla_{\perp} \mathscr{P}(\eta) \eta}{1+\left|\nabla_{\perp} \eta\right|^{2}}-\partial_{x} \mathscr{P}(\eta) \eta, \\
W_{y}=\frac{\eta_{x} \eta_{y}+\eta_{y} \nabla_{\perp} \eta \cdot \nabla_{\perp} \mathscr{P}(\eta) \eta}{1+\left|\nabla_{\perp} \eta\right|^{2}}-\partial_{y} \mathscr{P}(\eta) \eta, \\
W_{z}=-\frac{\eta_{x}+\nabla_{\perp} \eta \cdot \nabla_{\perp} \mathscr{P}(\eta) \eta}{1+\left|\nabla_{\perp} \eta\right|^{2}} .
\end{gathered}
$$

Using the above formulae, the kinematic and dynamic boundary conditions can be rewritten as follows:

$$
\begin{aligned}
\eta_{t} & =G(\eta) \xi \\
\xi_{t} & =\frac{1}{2\left(1+\left|\nabla_{\perp} \eta\right|^{2}\right)}\left[(G(\eta) \xi)^{2}\right. \\
& +2(G(\eta) \xi)\left(\nabla_{\perp} \eta \cdot \nabla_{\perp} \xi\right)-\left|\nabla_{\perp} \xi\right|^{2}\left|\nabla_{\perp} \eta\right|^{2} \\
& \left.+\left(\nabla_{\perp} \eta \cdot \nabla_{\perp} \xi\right)^{2}-\left|\nabla_{\perp} \xi\right|^{2}\right] \\
& +\frac{E}{2}\left[-\frac{\left(\eta_{x}+\nabla_{\perp} \eta \cdot \nabla_{\perp} \mathscr{P}(\eta) \eta\right)^{2}}{1+\left|\nabla_{\perp} \eta\right|^{2}}+2 \partial_{x} \mathscr{P}(\eta) \eta\right. \\
& \left.+\left|\nabla_{\perp} \mathscr{P}(\eta) \eta\right|^{2}\right]-\eta+B \nabla_{\perp} \cdot\left[\frac{\nabla_{\perp} \eta}{\sqrt{1+\left|\nabla_{\perp} \eta\right|^{2}}}\right] .
\end{aligned}
$$

Linearizing the initial system (18)-(19) and seeking solutions in the form $e^{i\left(k_{x} x+k_{y} y-\omega t\right)}$ lead to the dispersion relation

$$
\omega^{2}=|\mathbf{k}| \tanh (|\mathbf{k}|)\left(1+B|\mathbf{k}|^{2}\right)+\frac{E k_{x}^{2}}{2},
$$

where $\omega$ is the wave frequency and $\mathbf{k}=\left(k_{x}, k_{y}\right)$ is the wavenumber with its component corresponding to $x$ - and $y$ directions, respectively.

\section{Long-Wave Models}

Deriving long-wave models using the DNO was initiated by Craig and Groves [31]. It is well-known that the DNO is an analytical operator if the $C^{1}$-norm of $\eta$ is smaller than a constant. Therefore, the Taylor series of the DNO converge; namely, $G(\eta)$ can be expressed as $G(\eta)=\sum_{j=0}^{\infty} G_{j}(\eta)$, and the first three terms are given by

$$
\begin{aligned}
& G_{0}(\eta)=\left(-\Delta_{\perp}\right)^{1 / 2} \tanh \left(\left(-\Delta_{\perp}\right)^{1 / 2}\right), \\
& G_{1}(\eta)=-G_{0} \eta G_{0}-\nabla_{\perp} \cdot \eta \nabla_{\perp}, \\
& G_{2}(\eta)=\frac{1}{2} \Delta_{\perp} \eta^{2} G_{0}+\frac{1}{2} G_{0} \eta^{2} \Delta_{\perp}+G_{0} \eta G_{0} \eta G_{0} .
\end{aligned}
$$

It is worth mentioning that, in contrast to [32], we expand the DNO in this paper based on the results of Nicholls and Reitich [33], who provided the joint analyticity and analytic continuation results for the DNO. We assume $L_{x}$ is the typical length scale in $x$-direction. Since the bottom of the fluid is at $z=-1$, we define $\delta \triangleq 1 / L_{x} \ll 1$. If we consider the isotropic problem; namely, the typical wavelength in $y$-direction, $L_{y}$, is similar to $L_{x}$; therefore, $\left(-\Delta_{\perp}\right)^{1 / 2}=O(\delta)$ and we can expand

$$
\begin{aligned}
\tanh \left(\left(-\Delta_{\perp}\right)^{1 / 2}\right)= & \left(-\Delta_{\perp}\right)^{1 / 2}-\frac{1}{3}\left(-\Delta_{\perp}\right)^{3 / 2} \\
& +\frac{2}{15}\left(-\Delta_{\perp}\right)^{5 / 2}+O\left(\delta^{7}\right) .
\end{aligned}
$$

Following [34], we can also expend $\mathscr{P}$ as

$$
\begin{aligned}
\mathscr{P}(\eta)= & G^{-1}(\eta) \partial_{x} \\
= & \left(G_{0}+G_{1}(\eta)+G_{2}(\eta)+\cdots\right)^{-1} \partial_{x} \\
= & \left(1+G_{0}^{-1} G_{1}+G_{0}^{-1} G_{2}+\cdots\right)^{-1} G_{0}^{-1} \partial_{x} \\
= & G_{0}^{-1} \partial_{x}-G_{0}^{-1} G_{1} G_{0}^{-1} \partial_{x}-G_{0}^{-1} G_{2} G_{0}^{-1} \partial_{x} \\
& +G_{0}^{-1} G_{1} G_{0}^{-1} G_{1} G_{0}^{-1} \partial_{x}+\text { h.o.t. }
\end{aligned}
$$

In the meantime, we obtain

$$
\begin{aligned}
G_{0}^{-1} & =\left[-\Delta_{\perp}-\frac{1}{3} \Delta_{\perp}^{2}-\frac{2}{15} \Delta_{\perp}^{3}+O\left(\delta^{8}\right)\right]^{-1} \\
& =\left(-\Delta_{\perp}\right)^{-1}+\frac{1}{3}+\frac{1}{45} \Delta_{\perp}+O\left(\delta^{4}\right) .
\end{aligned}
$$

4.1. Benney-Luke Scaling. The isotropic Boussinesq regime includes the additional expectation of small amplitude motions. First of all we assume $E=O\left(\delta^{2}\right)$ and the Bond number $B=O(1)$. The scales for the variables are as follows: $L_{x}=O(1 / \delta), L_{y}=O(1 / \delta)$; the time scale is of order $O(1 / \delta)$, $\eta=O\left(\delta^{2}\right)$ and $\xi=O(\delta)$. It follows that

$$
\begin{aligned}
& G(\eta)=-\Delta_{\perp}-\frac{1}{3} \Delta_{\perp}^{2}-\nabla_{\perp} \cdot \eta \nabla_{\perp}+\text { h.o.t., } \\
& \mathscr{P}(\eta)=-\Delta_{\perp}^{-1} \partial_{x}+\text { h.o.t. }
\end{aligned}
$$

We proceed by expanding (18)-(19) in terms of the small parameter $\delta$ and truncating at $O\left(\delta^{5}\right)$

$$
\begin{aligned}
& \eta_{t}=-\Delta_{\perp} \xi-\frac{1}{3} \Delta_{\perp}^{2} \xi-\nabla_{\perp} \cdot \eta \nabla_{\perp} \xi \\
& \xi_{t}=-\frac{1}{2}\left|\nabla_{\perp} \xi\right|^{2}-E \partial_{x} \Delta_{\perp}^{-1} \partial_{x} \eta-\eta+B \Delta_{\perp} \eta .
\end{aligned}
$$


Taking derivative with respect to time for (27) yields

$$
\xi_{t t}=-\frac{1}{2}\left(\left|\nabla_{\perp} \xi\right|^{2}\right)_{t}-E \partial_{x} \Delta_{\perp}^{-1} \partial_{x} \eta_{t}-\eta_{t}+B \Delta_{\perp} \eta_{t}
$$

Substituting (26) into (28) and retaining terms valid to $O\left(\delta^{5}\right)$ yield

$$
\begin{aligned}
\xi_{t t}= & -\frac{1}{2}\left(\left|\nabla_{\perp} \xi\right|^{2}\right)_{t}+E \partial_{x x} \xi+\Delta_{\perp} \xi+\frac{1}{3} \Delta_{\perp}^{2} \xi+\nabla_{\perp} \\
& \cdot \eta \nabla_{\perp} \xi-B \Delta_{\perp}^{2} \xi .
\end{aligned}
$$

Equation (27) implies that $\xi_{t}=-\eta+$ h.o.t.; therefore, substituting (29) yields

$$
\begin{gathered}
\xi_{t t}-\Delta_{\perp} \xi-E \partial_{x}^{2} \xi+\left(B-\frac{1}{3}\right) \Delta_{\perp}^{2} \xi+\left(\left|\nabla_{\perp} \xi\right|^{2}\right)_{t} \\
+\xi_{t} \Delta_{\perp} \xi=0 .
\end{gathered}
$$

If the original problem is independent of the transverse direction, (27) reduces to

$$
\xi_{t t}-(1+E) \xi_{x x}+\left(B-\frac{1}{3}\right) \xi_{x x x x}+\left(\xi_{x}^{2}\right)_{t}+\xi_{t} \xi_{x x}=0
$$

And its traveling-wave solutions satisfy

$$
\left(c^{2}-1-E\right) \xi_{x x}+\left(B-\frac{1}{3}\right) \xi_{x x x x}-\frac{3 c}{2}\left(\xi_{x}^{2}\right)_{x}=0
$$

which admits KdV soliton

$$
\xi_{x}=-\frac{1+E-c^{2}}{c} \operatorname{sech}^{2}\left(\frac{x}{2} \sqrt{\frac{1+E-c^{2}}{B-1 / 3}}\right) .
$$

4.2. Kadomtsev-Petviashvili Scaling. If the variation in the $y$ direction is much slower than that in the $x$-direction, we choose $L_{y}=O\left(1 / \delta^{2}\right)$ and the scaling of the other variables is the same as the case of Benney-Luke. In the same vein, it is not difficult to obtain the following two equations:

$$
\begin{aligned}
& \eta_{t}=-\Delta \xi-\frac{1}{3} \xi_{x x x x}-\left(\eta \xi_{x}\right)_{x} \\
& \xi_{t}=-\frac{1}{2} \xi_{x}^{2}-(1+E) \eta+B \eta_{x x}
\end{aligned}
$$

Following the same argument stated in Section 4.1, we eliminate $\eta$ and arrive at

$$
\begin{aligned}
\xi_{t t} & -\Delta_{\perp} \xi-E \xi_{x x}+\left(B-\frac{1}{3}\right) \xi_{x x x x}+\left(\xi_{x}^{2}\right)_{t}+\xi_{t} \xi_{x x} \\
& =0 .
\end{aligned}
$$

We change variables as

$$
\begin{aligned}
& X=\delta x-\delta t, \\
& T=\delta^{3} t \\
& Y=\delta^{2} y \Longrightarrow \\
& \partial_{t}=-\delta \partial_{X}+\delta^{3} \partial_{T}, \\
& \partial_{x}=\delta \partial_{X}, \\
& \partial_{y}=\delta^{2} \partial_{Y} .
\end{aligned}
$$

Therefore, we can recast (35) as

$$
\begin{aligned}
& \left(-\delta \partial_{X}+\delta^{3} \partial_{T}\right)^{2} \xi-\left(\delta^{2} \partial_{X X}+\delta^{4} \partial_{Y Y}\right) \xi-E \delta^{2} \xi_{X X} \\
& +\left(B-\frac{1}{3}\right) \delta^{4} \xi_{X X X X}+\left(-\delta \partial_{X}+\delta^{3} \partial_{T}\right) \delta^{2}\left(\xi_{X}\right)^{2} \\
& \quad+\xi_{X X}\left(-\delta \partial_{X}+\delta^{3} \partial_{T}\right) \delta^{2} \xi=0 .
\end{aligned}
$$

Truncating at $O\left(\delta^{4}\right)$ and returning back to the original scale, we arrive at

$$
\begin{aligned}
& 2 \xi_{t x}+(2+E) \xi_{x x}+\left(\frac{1}{3}-B\right) \xi_{x x x x}+\xi_{y y}+3 \xi_{x} \xi_{x x} \\
& \quad=0 .
\end{aligned}
$$

Taking derivative with respect to $x$ and letting $f=\xi_{x}$, the KP equation takes the form

$$
\begin{aligned}
& {\left[2 f_{t}+(2+E) f_{x}+\left(\frac{1}{3}-B\right) f_{x x x}+3 f f_{x}\right]_{x}+f_{y y}} \\
& \quad=0 .
\end{aligned}
$$

4.3. Lumps. Fully confined three-dimensional traveling waves, known as lumps, do not exist in pure gravity waves ([35]); however, if the surface tension is also included and strong enough, lumps do exist and were first predicted by the celebrated KP equation. Equation (39) is the KP equation whose coefficient of the $f_{x}$ term is modified by the electric field, and it admits analytical fully confined traveling-wave solutions, which is called the "lumps" in the literature. Pego and Quintero [36] mathematically proved the existence of lumps in the Benney-Luke equation (30). In the present paper, we numerically search for these solutions in (30). We assume $\xi$ translates with speed $c$ in the $x$-direction, namely, $\xi(x-c t, y)$; therefore, (30) becomes

$$
\begin{aligned}
& \left(c^{2}-E\right) \xi_{x x}-\Delta_{\perp} \xi+\left(B-\frac{1}{3}\right) \Delta_{\perp}^{2} \xi-c\left(\left|\nabla_{\perp} \xi\right|^{2}\right)_{x} \\
& -c \xi_{x} \Delta_{\perp} \xi=0 .
\end{aligned}
$$

The numerical scheme adopted to find these lump-type solutions was first proposed by Petviashvili [37], and here we follow the procedure given by Wang [8]. Applying the Fourier transform to (40) leads to

$$
\widehat{\xi}=\frac{c\left(i k_{x} \widehat{|\nabla \xi|^{2}}+\widehat{\xi_{x} \Delta \xi}\right)}{\left(E-c^{2}\right) k_{x}^{2}+|\mathbf{k}|^{2}+(B-1 / 3)|\mathbf{k}|^{4}} \triangleq \mathscr{M}[\widehat{\xi}],
$$




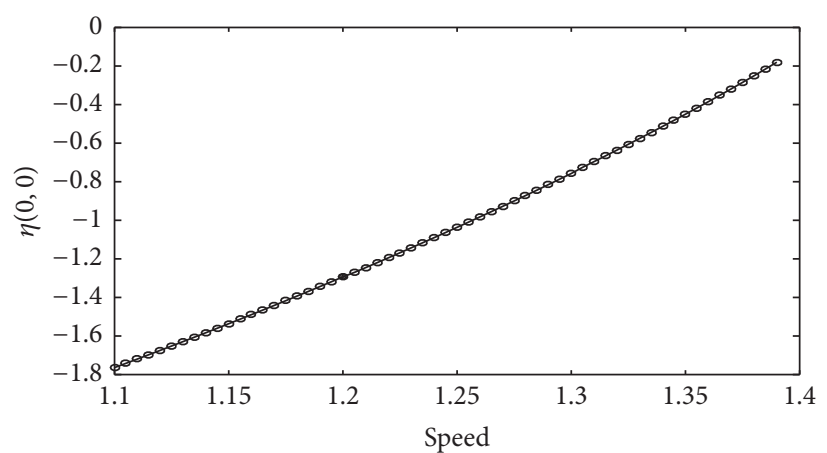

(a)

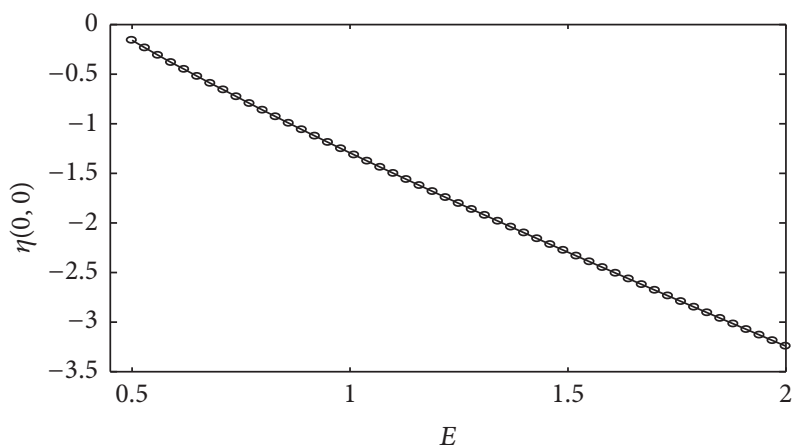

(b)

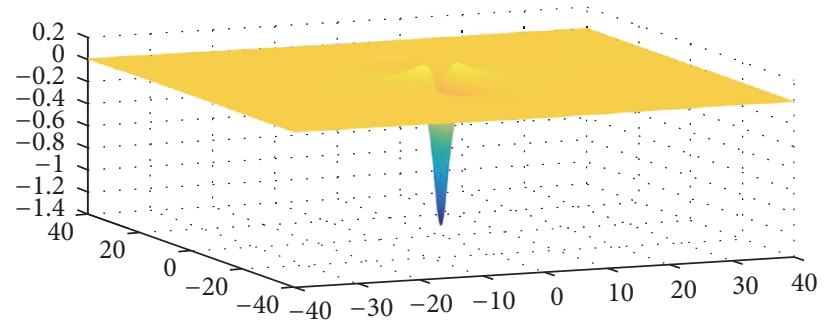

(c)

Figure 2: (a) Speed-amplitude bifurcation diagram of lumps in (30) for $E=1$ and $B=2 / 3$, which is monotonically increasing. (b) $E$ amplitude bifurcation diagram of lumps in (30) for $c=1.2$ and $B=2 / 3$, which is monotonically decreasing. (c) A typical depression wave profile $(c=1.2$ and $\eta(0,0)=-1.2934)$ which is labeled by a solid dark point in (a).

where $\mathbf{k}=\left(k_{x}, k_{y}\right)$ is the vector of wavenumber. Following [8], we propose the numerical iteration scheme as

$$
\widehat{\xi}_{n+1}=\alpha_{n} \mathscr{M}\left[\widehat{\xi}_{n}\right]
$$

where $\alpha_{n}$ is defined as

$$
\alpha_{n}=\frac{\iint\left|\widehat{\xi}_{n}\right|^{2} d k_{x} d k_{y}}{\iint \widehat{\xi}_{n}^{*} \mathscr{M}\left[\hat{\xi}_{n}\right] d k_{x} d k_{y}} .
$$

In Figure 2 we present the bifurcation diagram and typical profile of lumps in (30) for $B=2 / 3$. Figure 2(a) shows the speed-amplitude curve for fixed $E=1$, and Figure 2(b) shows the $E$-amplitude curve for fixed $c=1.2$, both of which are monotonic. For positive $c$, only depression lumps which feature a negative value at the center (see Figure 2(c)) were found.

For time-dependent simulation of the Benney-Luke equation (30), we introduce a new variable $\theta \triangleq \xi_{t}$; then it follows that

$$
\begin{aligned}
\theta_{t}= & \Delta_{\perp} \xi+E \xi_{x x}-\left(B-\frac{1}{3}\right) \Delta_{\perp}^{2} \xi-2 \nabla_{\perp} \xi \cdot \nabla_{\perp} \theta \\
& -\theta \Delta_{\perp} \xi \\
\xi_{t}= & \theta,
\end{aligned}
$$

which is a first-order dispersive system and can be integrated using pseudo-spectral method (see, e.g., $[8,15,38,39]$ ). We study the transverse instability of plane solitary-wave solutions (33), which gives mechanics of generation of lumps.
We denote a plane solution (33) by $\mathscr{K}$ and perturb it using a long wave in the transverse direction; namely,

$$
\xi(x, y, 0)=\mathscr{K}(x)\left[1+0.01 \cos \left(\frac{\pi y}{30}\right)\right]
$$

is taken as the initial profile. The subsequent evolution of this initial data shows a focusing behaviour which is arrested by the formation of a lump propagating almost steadily (the snapshots are shown in Figure 3).

\section{Conclusion}

In the present paper, we have studied the electro-capillarygravity waves with a horizontal electric field. For simplicity, we make assumption that the permittivity of the fluid is much larger than that of the upper-layer gas. Therefore, as the firstorder approximation, the whole system could be reduced to a one-layer problem. The Dirichlet-Neumann operator, which transfers the Dirichlet boundary condition to the Neumann derivative on the boundary via solving the Laplace equation, plays an important role in this problem. Based on the longwave approximation and symbolical calculations, we have derived the unidirectional KP-like model and bidirectional Benney-Luke-like model. Then the bifurcation diagram and typical profiles for three-dimensional solitary waves (i.e., lumps) were found in the modified Benney-Luke equation based on the Petviashvili method.

Very recently considerable attention has been paid to asymmetric gravity-capillary waves. Wang et al. [40] first found asymmetric plane solitary waves in the full Euler equations in [40] and asymmetric lumps in a model equation 


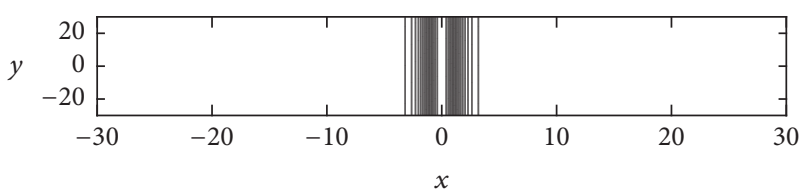

(a)

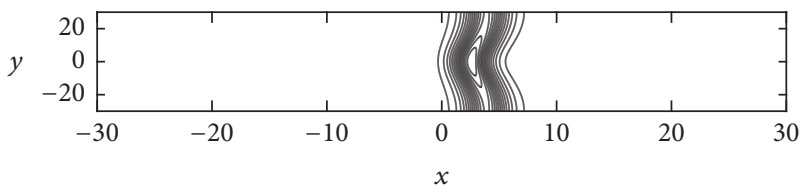

(b)

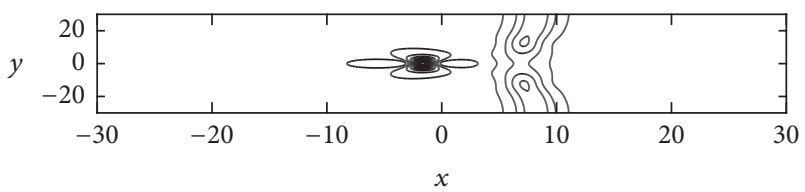

(c)

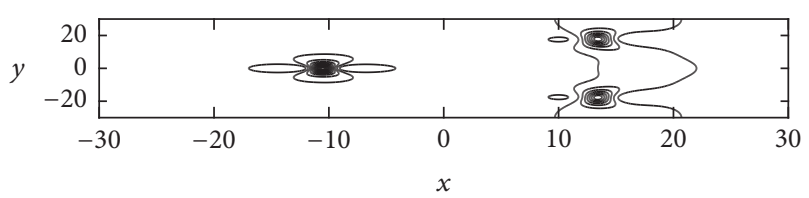

(d)

FIGURE 3: Time evolution of a plane solitary wave in the presence of transverse perturbation (the specific form of the perturbation is given in (45)) for $t=0,140,220,300$ (from (a) to (d)).

in [41]. Shimizu and Shoji [42] and Gao et al. [43] thoroughly investigated asymmetric periodic waves. Gao et al. [44] found asymmetric generalized solitary waves in the full Euler equations. These findings raise the question as to the existence and stability of asymmetric waves in electrified waves, which will be left for future studies.

Finally we remark that if the electric field is strong enough so that gravity and surface tension are negligible, linearizing the original system yields

$$
\begin{aligned}
\phi_{t} & =-E W_{x}, \\
W_{z} & =-\eta_{x}, \\
\eta_{t} & =\phi_{z} .
\end{aligned}
$$

Combining the above equations, we end up with a wave equation $\eta_{t t}-E \eta_{x x}=0$ for arbitrary depth of fluid, which is commonly true in shallow water only in water-wave problems.

\section{Conflicts of Interest}

The author declares that there are no conflicts of interest regarding the publication of this paper.

\section{References}

[1] E. M. Griffing, S. George Bankoff, M. J. Miksis, and R. A. Schluter, "Electrohydrodynamics of thin flowing films," Journal of Fluids Engineering, vol. 128, no. 2, pp. 276-283, 2006.
[2] Z. Lin, Y. Zhu, and Z. Wang, "Local bifurcation of electrohydrodynamic waves on a conducting fluid," Physics of Fluids, vol. 29, Article ID 032107, 2017.

[3] J. R. Melcher, "Electrohydrodynamic and magnetohydrodynamic surface waves and instabilities," Physics of Fluids, vol. 4, pp. 1348-1354, 1961.

[4] J. R. Melcher and G. I. Taylor, "Electrohydrodynamics: A review of the role of interfacial shear stresses," Annual Review of Fluid Mechanics, pp. 111-146, 1969.

[5] D. T. Papageorgiou and J.-M. Vanden-Broeck, "Large-amplitude capillary waves in electrified fluid sheets," Journal of Fluid Mechanics, no. 508, pp. 71-88, 2004.

[6] G. I. Taylor, "Disintegration of water droplets in an electric field," Proceedings of the Royal Society of London A, vol. 280, pp. 383-397, 1964.

[7] B. S. Tilley, P. G. Petropoulos, and D. T. Papageorgiou, "Dynamics and rupture of planar electrified liquid sheets," Physics of Fluids, vol. 13, no. 12, pp. 3547-3563, 2001.

[8] Z. Wang, "Modelling nonlinear electrohydrodynamic surface waves over three-dimensional conducting fluids," Proceedings A, vol. 473, no. 2200, 20160817, 20 pages, 2017.

[9] N. Wu and W. B. Russel, "Electrohydrodynamic instability of dielectric bilayers: kinetics and thermodynamics," Industrial \& Engineering Chemistry Research, vol. 45, pp. 5455-5465, 2006.

[10] N. M. Zubarev, "Nonlinear waves on the surface of a dielectric liquid in a strong tangential electric field," Physics Letters A, vol. 333, no. 3-4, pp. 284-288, 2004.

[11] A. Gonzalez and A. Castellanos, "Nonlinear electrohydrodynamic waves on films falling down an inclined plane," Physical Review E, vol. 53, pp. 3573-3578, 1996.

[12] A. Miyara, "Numerical analysis on flow dynamics and heat transfer of falling liquid films with interfacial waves," Wärmeund Stoffübertragung, vol. 35, no. 4, pp. 298-306, 1999.

[13] G. I. Taylor and A. D. McEwan, "The stability of a horizontal fluid interface in a vertical electric field," Journal of Fluid Mechanics, vol. 22, p. 15, 1965.

[14] I. N. Aliev and S. O. Yurchenko, "Nonlinear waves propagating over a conducting ideal fluid surface in an electric field," Journal of Fluid Dynamics, vol. 44, no. 5, pp. 748-758, 2009.

[15] L. L. Barannyk, D. T. Papageorgiou, and P. G. Petropoulos, "Suppression of Rayleigh-Taylor instability using electric fields," Mathematics and Computers in Simulation, vol. 82, no. 6, pp. 1008-1016, 2012.

[16] H. Gleeson, P. W. Hammerton, D. T. Papageorgiou, and J.-M. Vanden-Broeck, "A new application of the Korteweg-de Vries Benjamin-Ono equation in interfacial electrohydrodynamics," Physics of Fluids, vol. 19, Article ID 031703, 2007.

[17] P. W. Hammerton, "Existence of solitary travelling waves in interfacial electrohydrodynamics," Wave Motion, vol. 50, no. 4, pp. 676-686, 2013.

[18] M. J. Hunt, J.-M. Vanden-Broeck, D. T. Papageorgiou, and E. I. Parau, "Benjamin-Ono Kadomtsev-Petviashvili models in interfacial electrohydrodynamics," European Journal of Mechanics - B, pp. 459-463, 2017.

[19] N. M. Zubarev, "Nonlinear waves on the surface of a dielectric liquid in a horizontal electric field in 3D geometry: Exact solutions," JETP Letters, vol. 89, no. 6, pp. 271-274, 2009.

[20] N. M. Zubarev and O. V. Zubareva, "Nonlinear dispersion relation for electrocapillary waves on the surface of a dielectric liquid," Technical Physics Letters, vol. 32, pp. 1027-1029, 2006. 
[21] D. T. Papageorgiou, P. G. Petropoulos, and J.-M. VandenBroeck, "Gravity capillary waves in fluid layer under normal electric field," Physical Review E, vol. 72, Article ID 051601, 2005.

[22] B. Tao and D. L. Guo, "Fully nonlinear capillary-gravity wave patterns under tangential electric field," Computers \& Mathematics with Applications, vol. 67, pp. 627-635, 2014.

[23] C. V. Easwaran, "Solitary waves on a conducting fluid layer," Physics of Fluids, vol. 31, no. 11, pp. 3442-3443, 1988.

[24] H. Kalisch, "Derivation and comparison of model equations for interfacial capillary-gravity waves in deep water," Mathematics and Computers in Simulation, vol. 74, no. 2-3, pp. 168-178, 2007.

[25] H. Kalisch and J. L. Bona, "Models for internal waves in deep water," Discrete and Continuous Dynamical Systems, pp. 1-20, 2000.

[26] L. D. Landau and E. M. Lifshitz, "Course of Theoretical Physics," in Electrodynamics of Continuous Media, vol. 8, 1984.

[27] R. Coifman and Y. Meyer, "Nonlinear harmonic analysis and analytic dependence," in Proceedings of Symposia in Pure Mathematics, pp. 71-78, 1985.

[28] W. Craig, U. Schanz, and C. Sulem, “The modulational regime of three-dimensional water waves and the Davey-Stewartson system," Annales de l'Institut Henri Poincaré, vol. 14, no. 5, pp. 615-667, 1997.

[29] W. Craig and D. P. Nicholls, "Travelling two and three-dimensional capillary gravity water waves," SIAM Journal on Mathematical Analysis, vol. 32, no. 2, pp. 323-359, 2000.

[30] D. P. Nicholls and F. Reitich, "Stability of High-Order Perturbative Methods for the Computation of Dirichlet-Neumann Operators," Journal of Computational Physics, vol. 170, no. 1, pp. 276-298, 2001.

[31] W. Craig and M. Groves, "Hamiltonian long-wave approximations to the water-wave problem," Wave Motion, pp. 367-389, 1994.

[32] W. Craig and C. Sulem, "Numerical simulation of gravity waves," Journal of Computational Physics, vol. 108, pp. 73-83, 1993.

[33] D. P. Nicholls and F. Reitich, "Analytic continuation of DirichletNeumann operators," Numerische Mathematik, vol. 94, no. 1, pp. 107-146, 2003.

[34] Z. Wang, J.-M. Vanden-Broeck, and H. Meng, "A quasi-planar model for gravity-capillary interfacial waves in deep water," Studies in Applied Mathematics, vol. 133, no. 2, pp. 232-256, 2014.

[35] W. Craig, "Non-existence of solitary water waves in three dimensions," The Royal Society of London. Philosophical Transactions. Series A. Mathematical, Physical and Engineering Sciences, vol. 360, no. 1799, pp. 2127-2135, 2002.

[36] R. L. Pego and J. R. Quintero, “Two-dimensional solitary waves for a Benney-Luke equation," Physica D: Nonlinear Phenomena, vol. 132, no. 4, pp. 476-496, 1999.

[37] V. I. Petviashvili, "Equation of an extraordinary soliton," Soviet Journal of Plasma Physics, pp. 257-258, 1976.

[38] P. A. Milewski and Z. Wang, "Transversally periodic solitary gravity-capillary waves," Proceedings of the Royal Society of London A, pp. 2013-0537, 2014.

[39] Z. Wang and P. A. Milewski, "Dynamics of gravity-capillary solitary waves in deep water," Journal of Fluid Mechanics, vol. 708, pp. 480-501, 2012.

[40] Z. Wang, J.-M. Vanden-Broeck, and P. A. Milewski, "Asymmetric gravity-capillary solitary waves on deep water," Journal of Fluid Mechanics, vol. 759, p. R2, 2014.
[41] Z. Wang and J.-M. Vanden-Broeck, "Multilump symmetric and nonsymmetric gravity-capillary solitary waves in deep water," SIAM Journal on Applied Mathematics, vol. 75, no. 3, pp. 978998, 2015.

[42] C. Shimizu and M. Shoji, "Appearance and disappearance of non-symmetric progressive capillary-gravity waves of deep water," Japan Journal of Industrial and Applied Mathematics, vol. 29, no. 2, pp. 331-353, 2012.

[43] T. Gao, Z. Wang, and J.-M. Vanden-Broeck, "Investigation of symmetry breaking in periodic gravity-capillary waves," Journal of Fluid Mechanics, vol. 811, pp. 622-641, 2017.

[44] T. Gao, Z. Wang, and J.-M. Vanden-Broeck, "On asymmetric generalized solitary gravity-capillary waves in finite depth," Proceedings of the Royal Society A, vol. 472, no. 2194, 2016. 


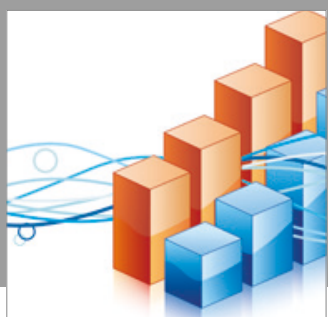

Advances in

Operations Research

vatersals

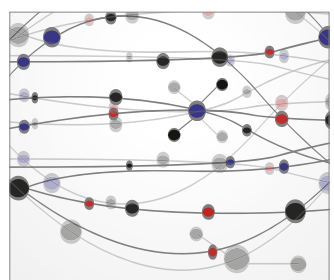

\section{The Scientific} World Journal
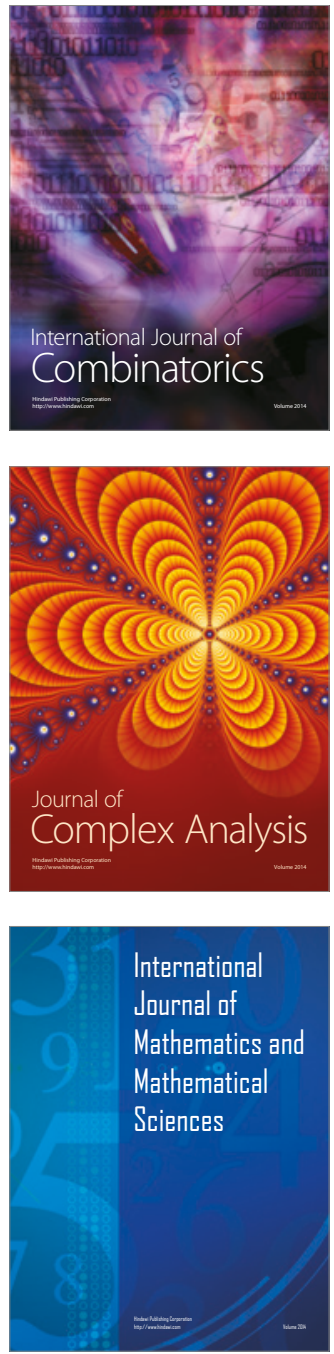
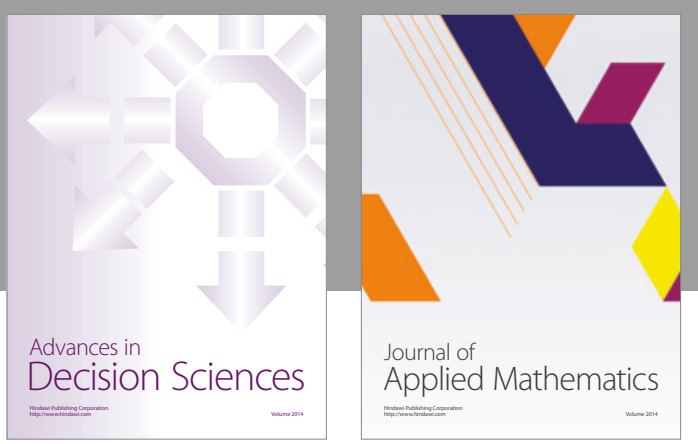

Algebra

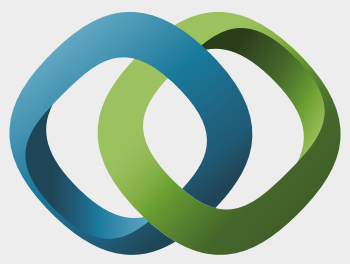

\section{Hindawi}

Submit your manuscripts at

https://www.hindawi.com
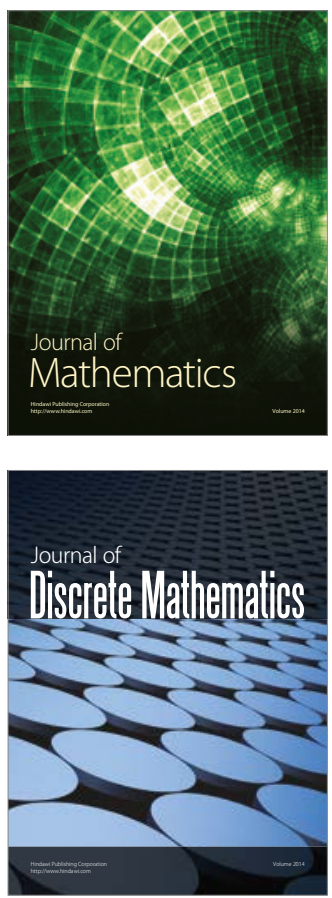

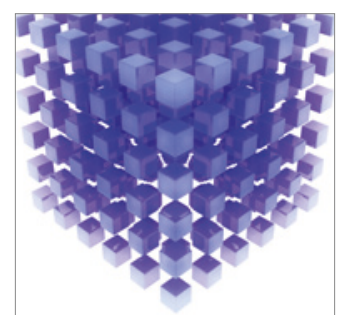

Mathematical Problems in Engineering
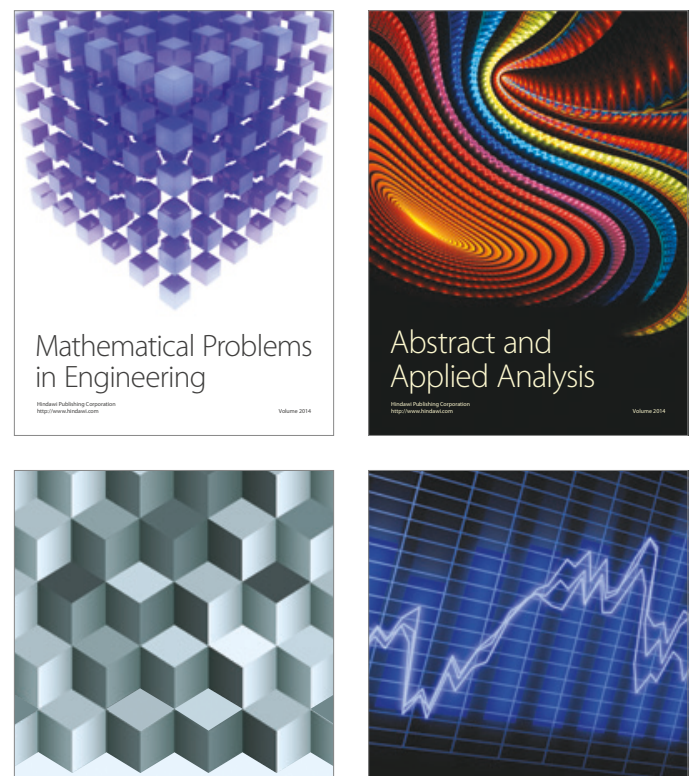

Journal of

Function Spaces

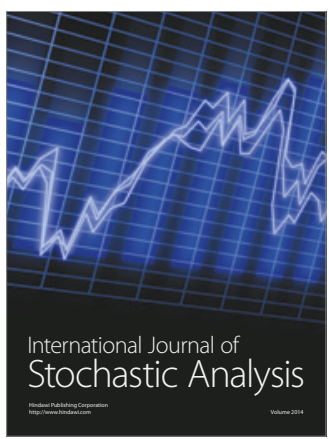

Probability and Statistics
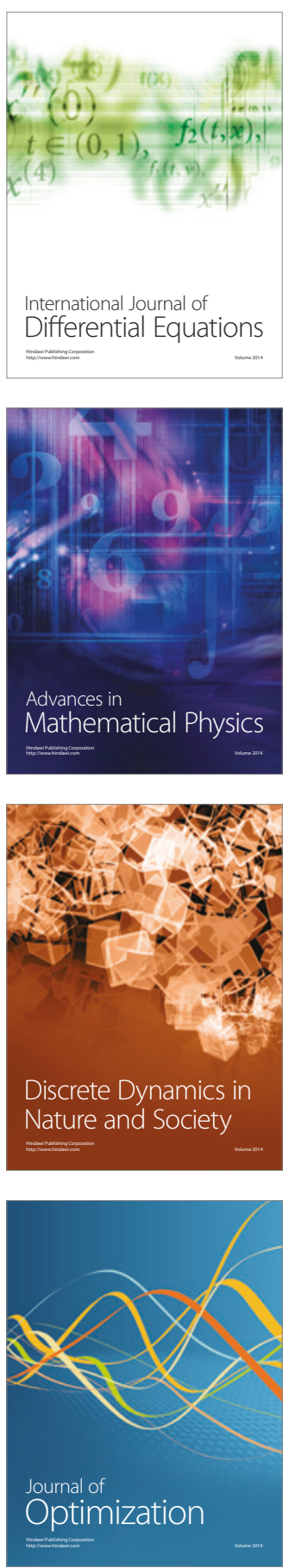